\title{
Peculiarities of Ga and Te incorporation in glassy arsenic selenides
}

\author{
R. Golovchak ${ }^{1}$, Ya. Shpotyuk ${ }^{2,3}$, C.M. Thomas ${ }^{1}$, V. Nazabal ${ }^{2}$, C. Boussard-Pledel ${ }^{2}$, B. Bureau ${ }^{2}$, H. \\ Jain $^{4}$ \\ ${ }^{1}$ Department of Physics \& Astronomy, Austin Peay State University \\ Clarksville, TN 37044, USA \\ ${ }^{2}$ Equipe Verres et Céramiques, UMR-CNRS 6226, Université de Rennes 1 \\ 35042 Rennes Cedex, FRANCE \\ ${ }^{3}$ Centre for Innovation and Transfer of Natural Sciences and Engineering Knowledge, \\ University of Rzeszow, Rzeszow, 35-959, POLAND \\ ${ }^{4}$ Department of Materials Science and Engineering, Lehigh University, Bethlehem, PA 18015-3195, USA \\ * Corresponding author: Tel.: + 1931 2216361, E-mail address: holovchakr@apsu.edu
}

\begin{abstract}
Effect of simultaneous $\mathrm{Ga}$ and Te addition on the structure of $\mathrm{As}_{2} \mathrm{Se}_{3}$ glasses is studied using Xray photoelectron spectroscopy (XPS), extended X-ray absorption fine structure (EXAFS) and Raman techniques. It is shown that most of As, Se and Te atoms build a covalent network according to their main valences. Three-fold coordinated As atoms form pyramidal structural units, which are connected via bridges of two-fold coordinated chalcogen atoms (Se, Te). On the other hand, coordination of Ga in glassy samples is found to be greater than three, as expected from its valence, increasing with Te content. Some of the As atoms appear to be converted into four-fold coordinated state at low Te concentration, while a fraction of Te and, possibly, Se atoms are thought to exist in a singly-coordinated (terminal) state in the vicinity of $\mathrm{Ga}$ in the samples with higher $\mathrm{Te}$ concentration.
\end{abstract}




\section{Introduction}

Tellurium-based glasses possess the widest infrared transmission window (including both atmospheric telecommunication windows 3-5 and 8-12 $\mu \mathrm{m}$ ) of all amorphous chalcogenides and are key to a number of applications in far-infrared optics, waveguiding for space telecommunication, optical and bio-sensing technologies [1-5]. However, tellurides themselves are not intrinsically good glass-formers [6,7] and require significant materials engineering for device fabrication. On the other hand, selenium-based glasses are known to have the widest glass-forming regions of all chalcogenides [6,7], but their transmission window is narrower due to a smaller mass of Se compared to Te, which determines the phonon energy spectrum of the amorphous network and, therefore, upper wavelength limit of transmission [8,9]. One of the strategies for stable IR glass engineering and high-quality IR optical fiber fabrication relies on combining the high transparency of tellurides with outstanding glass-forming ability of selenides. In this regard, the mixture of binary arsenic tellurides and selenides looks especially attractive due to the isomorphic structure of their main network-forming units (arsenic-based pyramids and chalcogen chains) [6,9-11]. As a proof of concept, Te-As-Se (TAS) glasses have been successfully engineered recently and some of them proposed for IR fiber applications [12,13].

Good solubility of Ga in these materials makes TAS also a prospective host matrix for rare earth elements doping [14], as they are proven to retain their spectroscopic characteristics with Ga addition better than most others [15]. This opens a range of possibilities for the application of rare earth doped TAS glasses in optical sensing, as active media for lasers, optical amplifiers and broad band sources in the mid-infrared spectral range $[16,17]$. Therefore, the peculiarities of Ga and Te incorporation into the As-Se glass network should be studied thoroughly. Recently, the influence of Ga on the network of $\mathrm{As}_{2} \mathrm{Se}_{3}$ glass was investigated [18]. On the basis of XPS and EXAFS analysis, it was shown that Ga enters the glass structure in 4-fold coordinated form as in some crystalline counterparts. At higher concentrations, $\mathrm{Ga}$ caused nucleation of $\mathrm{Ga}_{2} \mathrm{Se}_{3}$ crystallites and, hypothetically, conversion of certain number of As atoms into four-fold coordinated state at the interface between the nucleation sites and host amorphous matrix. The existence of Se-Se and Ga- 
As bonds in the structure of Ga-modified stoichiometric arsenic selenide is also plausible from these results [18]. On the other hand, excess of Te atoms beyond 30 at.\% in As-Se network is known to cause partial crystallization too [19]. Therefore, it is important to understand the role of Te in As-Se matrix and its influence on the Ga chemical state and crystallization ability. This can be accomplished through the investigations of As-Se glasses with different content of Te and fixed amount of Ga.

So, in the present work we have performed comprehensive structural studies of Te incorporation in binary As-Se glasses containing 2 at.\% of Ga, which form a model matrix for TAS doped with rare earth elements. A combination of high resolution X-ray photoelectron spectroscopy (XPS) and extended X-ray absorption fine-structure spectroscopy (EXAFS), which is proven to be effective for structural studies in chalcogenide glasses [20,21], has been used for these purposes. The analysis of core level XPS spectra is used to quantify the relative content of atoms in different chemical environments and oxidation states, whereas EXAFS is used as a complementary technique to determine local coordination number and distance around each kind of constituent atoms. Raman spectroscopy is used as additional tool to verify the assignment of XPS peak moieties to glassforming structural units.

\section{Experimental}

The $\mathrm{Ga}_{2}\left(\mathrm{As}_{0.4} \mathrm{Se}_{0.6}\right)_{98-\mathrm{x}} \mathrm{Te}_{\mathrm{x}}(\mathrm{x}=0,10,15,20,30)$ glasses were prepared by conventional meltquench route from high-purity chemical precursors, viz. Ga (99, 99999 \%), As (99, 999\%), Se (99, 999\%) and Te (99, 9999\%). As and Se were additionally purified by heating them to 310 and 240 ${ }^{\circ} \mathrm{C}$, respectively, and keeping at this temperature for $16 \mathrm{~h}$ under vacuum pump. Appropriate amounts of Ga, As, Se and Te (with total weight close to $30 \mathrm{~g}$ ) were vacuum sealed in silica tube of $10 \mathrm{~mm}$ diameter. Then, the ampoules were heated up to $900{ }^{\circ} \mathrm{C}$ with $2{ }^{\circ} \mathrm{C} / \mathrm{min}$ rate in a rocking furnace, homogenized for $10 \mathrm{~h}$ and quenched into water from $750{ }^{\circ} \mathrm{C}$. To relieve the mechanical strains introduced during rapid quenching, the samples were additionally annealed for $6 \mathrm{~h}$, at $\sim 10$ ${ }^{\circ} \mathrm{C}$ below the glass transition temperature determined by differential scanning calorimetry. All the 
obtained materials were in glassy form as established by the absence of crystalline reflections in Xray diffraction (XRD) patterns, except the $\mathrm{Ga}_{2}\left(\mathrm{As}_{0.4} \mathrm{Se}_{0.6}\right)_{68} \mathrm{Te}_{30}$ sample, which showed the presence of crystalline inclusions [19], and was included into the experimental set for comparison.

High resolution XPS spectra were recorded with Scienta ESCA-300 spectrometer (monochromatic $\mathrm{Al} K_{\alpha} \mathrm{X}$-rays) on the samples fractured in situ in the spectrometer's measurement chamber under a vacuum of $2 \times 10^{-8}$ Torr or better. For all measurements the angle between the surface and detector was $90^{\circ}$. The instrument was operated in a mode that yielded a Fermi-level width of $0.4 \mathrm{eV}$ for $\mathrm{Ag}$ metal and at a full width at half maximum (FWHM) of $0.54 \mathrm{eV}$ for $\mathrm{Ag} 3 d_{5 / 2}$ core level peak. Energy scale was calibrated using the Fermi level of clean Ag. The XPS data consisted of survey scans over the entire binding energy (BE) range and selected scans over the valence band or core level photoelectron peaks of interest. Surface charging from the photoelectron emission was neutralized using a low energy $(<10 \mathrm{eV})$ electron flood gun. The experimental positions of the valence band and core levels for all of the investigated samples were adjusted by referencing to the $4 f_{7 / 2}$ core level peak of pure $\mathrm{Au}$ at $84.0 \mathrm{eV}$, as described elsewhere [22].

Data analysis was conducted with standard CASA-XPS software package. For analyzing the core-level spectra, Shirley background was subtracted and a Voigt line shape was assumed for the peaks [23]. The $3 d$ core-level XPS spectra of Ga, As, Se and $4 d$ of Te were used for quantitative analysis of chemical order in the investigated samples. The number of doublets (each consisting of $d_{5 / 2}$ and $d_{3 / 2}$ components owing to a spin-orbit splitting) within a given peak was determined by an iterative curve fitting procedure in which a doublet was added only if it significantly improved the goodness of the fit. The parameters used to link the $d_{5 / 2}$ and $d_{3 / 2}$ components were: a peak separation of $0.46 \mathrm{eV}$ for $\mathrm{Ga}, 0.70 \mathrm{eV}$ for As, $0.85 \mathrm{eV}$ for Se and $1.46 \mathrm{eV}$ for Te, and an area ratio of 1.4 for all of the considered doublets belonging to a given $d$ core level. During fitting of data, the FWHM was assumed to be the same for the peaks within one doublet, but different FWHM values were allowed for separate doublets of the same core-level peak. The mix between the Gaussian and Lorentzian in the Voigt function was chosen to be the same for all doublets of a given core-level. The 
uncertainties in the peak position and area of each component were $\pm 0.05 \mathrm{eV}$ and $\pm 2 \%$ respectively.

No elements other than the glass components were observed in the survey XPS spectra of fractured surfaces, which showed only peaks associated with the Ga, As, Se and Te core levels and related Auger lines identified using the reference spectra in the PHI handbook [24]. In particular, there was no observable evidence of oxygen or sulfur contaminations on any of the fractured surfaces.

Coordination numbers and interatomic distances around each kind of constituent atoms were determined by EXAFS technique. These measurements were performed at X18B X-ray beam-line at National Synchrotron Light Source, Brookhaven National Laboratory. The samples were powdered and glued onto a 'Kapton' tape. Data collection was performed in transmission detection mode at the K-edges of Ga (10.367 keV), As (11.863 keV), Se (12.658 keV) and Te (31.814 keV) using sealed ion chambers of Oxford Danfysic filled with appropriate gas mixture. The energy scale was calibrated using pure Se and Te as standards, ensuring an experimental accuracy in energy of about $1 \mathrm{eV}$. The EXAFS signal presents modulation in the absorption coefficient $\mu_{i}$ as a function of X-ray energy $E=\hbar \omega$. Using one-electron approximation of Fermi's Golden Rule for $\mu_{i}(\omega)$, under the plane wave approximation, the EXAFS equation for isotropic materials like glass can be expressed as [25]:

$$
\mu_{i}(k)=\frac{\mu_{1}(k)}{\mu_{0}(k)}=\sum_{j} \frac{n_{j} S_{0 j}^{2}(k) F_{j}(k) \cdot \sin \left(2 k R_{j}+\phi_{j}(k)\right)}{k R_{j}^{2} \cdot \exp \left(\frac{2 R_{j}}{\Lambda}+2 k^{2} \sigma_{0 j}^{2}\right)},
$$

where $\mu_{1}(k)$ and $\mu_{0}(k)$ are the terms contributed by single scattering and background, respectively (the multiple scattering terms are negligible for EXAFS region), $k \approx 0.512\left(E-E_{0}\right)^{1 / 2} \AA^{-1}$ is the wavevector of photoelectron, $E_{0}$ is the threshold energy, $S_{0 j}$ is the passive electron reduction factor, $n_{j}$ is the degeneracy of the path, $N_{j}=n_{j} S_{0 j}^{2}(k)$ is the number of neighbours in the $j^{\text {th }}$ shell at average distance $R_{j}, F_{j}(k)$ is the effective amplitude of backscattered electron wave, $\phi_{j}(k)$ is the 
effective phase shift between backscattered and outgoing electron wave, $\Lambda$ is the mean free path of photoelectrons and $\sigma_{0}^{2}$ is the Debye-Waller factor related with disorder.

The obtained EXAFS spectra were analysed with Athena-Artemis software package [26], the Kaiser-Bessel type of window function being applied to restrict the EXAFS data in $k$ space. Crystallographic data for $\mathrm{As}_{2} \mathrm{Se}_{3}$ [27] and $\mathrm{Ga}_{2} \mathrm{Se}_{3}$ [28] were used as input for FEFF calculations to obtain information on $F_{j}(k)$ and $\phi_{j}(k)$ for As, Se and Ga atoms, while 'Quick first shell theory' in Artemis software was used to generate an input file to calculate FEFF for Te atoms. The calculated scattering paths for nearest neighbours were used to fit first shell in $R$-space, using LevenbergMarquardt method of nonlinear least-squares minimization implemented in Artemis (the energy shift $\Delta E_{0}$ was fixed during fitting).

Raman spectroscopy data were measured in 80-550 $\mathrm{cm}^{-1}$ range with Horiba Xplora confocal microscope ( $\times 100$ objective), using $785 \mathrm{~nm}$ laser for excitation. The acquisition time was in the range of $100-200 \mathrm{~s}$ and the calculated power of excitation beam was $\sim 0.07 \mathrm{~mW} \cdot \mu \mathrm{m}^{-2}-$ low enough to avoid photo-induced crystallization proper to these materials under the above laser wavelength. Spectra collected from four different regions within fresh surface of each sample were averaged to increase the confidence in the data.

\section{Results and discussion}

Valence band XPS spectra of the investigated samples and the reference glasses are shown in Figure 1. The observed features are typical for the valence band of chalcogenide glasses [29]. Accordingly, the well-defined contribution at about $2 \mathrm{eV}$ is attributed to the lone pair ( $l p$ ) Se $4 p$ and Te $5 p$ electrons; the peak at about $5 \mathrm{eV}$ is ascribed to the $4 p$ and $5 p$ bonding states of Se and Te, respectively; the broad band at 7-16 eV is due to the overlap of signals from Ga $4 s$, As $4 s$, Se $4 s$ and Te 5 s electrons. At the same time, the valley at $\sim 3 \mathrm{eV}$, which is a characteristic of the Se-rich arsenic selenides [29], is not observed in the investigated glasses due to the broadening of Se $4 p$ and 
Te $5 p$ bonding state peaks by a prevailed concentration of chalcogen-As( $\mathrm{Ga}$ ) bonds (As $4 p$ and $\mathrm{Ga}$ $4 p$ bonding states give peaks in this range of BEs) [24,29].

The quantitative analysis of structural moieties can be accomplished through the analysis of $d$ electron core level XPS spectra for constituting chemical elements, which are proven to be highly sensitive to the short-range order in this kind of materials [18,20-22]. Such analysis is based on the difference in their electronegativity, which introduces specific chemical shifts in XPS signals from $d$ core-level electrons of Ga, As, Se and Te for different structural fragments, depending on the electron density distribution. These shifts cause the appearance of separate doublets in the fit of experimental XPS spectra, each of such doublets corresponding to a specific chemical environment (structural fragment) of the absorbing atom. For most of chemical elements, in general the higher is the electronegativity value of neighbours in the structural fragment or the oxidation number/coordination of the absorbing atom - the greater should be the shift of corresponding doublet to high-BE values. Thus, a number of doublets in the fit gives a number of possible chemical environments for the absorbing atom, while the area under each doublet gives the concentration of the corresponding moiety. The electronegativity values for Ga, As, Se and Te in the investigated samples are $\chi_{G a}=1.81, \chi_{A s}=2.18, \chi_{S e}=2.55$ and $\chi_{T e}=2.10$, respectively [30]. So, doublets corresponding to different chemical environments should be relatively well resolved in our XPS spectra, except for the difficulty in differentiating the As and Te neighbours in target atom environment because of their close electronegativities.

Compositional dependence of Ga $3 d$, Se $3 d$, As $3 d$ and Te $5 d$ core levels and their best fit analyses are presented in Figs. 2-4, respectively. Fitting parameters, such as peak position or BE ( $d_{5 / 2}$ components), the area $(A)$ and FWHM are given in Tables 1-3. For the identification of structural fragments we have used our earlier results for binary As-Se glasses [30], Ga-modified AsSe alloys [18] and complementary measured EXAFS data (Table 4, Fig. 5). As it was shown previously, the $\mathrm{As}_{2} \mathrm{Se}_{3}$ sample contains mostly As-Se-As and Se-As $<(\mathrm{Se})_{2}$ structural fragments (where the probed by XPS element is identified in bold font) [22,31]. Some deviations are possible in the form of "wrong” Se-Se and As-As homopolar bonds as a result of non-optimal synthesis 
conditions, but usually no more than a couple of percent of total number of bonds [32-34]. So it is compelling to assign the doublet observed in Se $3 d$ core level XPS spectrum of $\mathrm{As}_{2} \mathrm{Se}_{3}$ sample at $\sim 54.1 \mathrm{eV}\left(3 d_{5 / 2}\right.$ component, Se-I in Table 1$)$ to the As-Se-As structural fragments, and the one at $\sim 42.2 \mathrm{eV}$ in As $3 d$ core level XPS spectra to the Se-As $<(\mathrm{Se})_{2}$ environment (As-II in Table 2) in good agreement with the results obtained previously [22,31]. Introducing 2 at.\% of Ga into $\mathrm{As}_{2} \mathrm{Se}_{3}$ network leads to the appearance of additional high-BE (As-I) and low-BE (As-IV, Se-II) peaks (relative to the positions of the above structural fragments) in the fitting of As and Se $3 d$ core level spectra (see Figs. 2,3), as shown earlier [18]. The low-BE doublets in As and Se $3 d$ core level XPS spectra (Se-II and As-IV, Tables 1,2) are explained by the changes in the chemical environment around the absorbing As or Se atoms. Indeed, the addition of Ga leads to a substitution of As in some As-Se-As fragments with Ga which has lower electronegativity than As [30], and, therefore, causes the appearance of additional doublet at low-BE side $(\sim 53.3 \mathrm{eV})$ of Se $3 d$ core level XPS spectrum (Se-II in Table 1). Appearance of metal-metal bonds in the structure of Ga-containing chalcogenide glasses due to a substitution of Se in $\mathrm{AsSe}_{3 / 2}$ pyramids with As or Ga atoms (e.g. (As, Ga) $-\mathbf{A s}<(\mathrm{Se})_{2}$ and Se-As $<(\mathrm{As}, \mathrm{Ga})_{2}$ structural fragments) is a reason for the additional doublet observed at the low-BE side of As $3 d$ core level XPS spectra at $41.7 \mathrm{eV}$ (As-IV in Table 2) and higher FWHM of Ga 3d core level XPS peak (Ga-I in Table 3) [18]. The high-BE peak in As 3d core level XPS spectra at $\sim 42.4 \mathrm{eV}$ (As-I in Table 2) may be consistent with As atoms converted into the four-fold coordinated state (the average local coordination $~ 3.2$ in Table 4, determined from EXAFS at As K-edge [18], supports such an assumption) by the presence of Ga atoms in their vicinity, which also were confirmed to be four-fold coordinated according to EXAFS data [18].

In $\mathrm{Ga}_{2} \mathrm{As}_{35.2} \mathrm{Se}_{52.8} \mathrm{Te}_{10}$ glass the high-BE peak at $\sim 42.3 \mathrm{eV}$ in As $3 d$ core level XPS spectrum is still observed (Table 2), which, however, should be a superposition of four-fold coordinated As atoms and pure Se-As $<(\mathrm{Se})_{2}$ pyramids owing to its intermediate BE position in comparison to Tefree $\mathrm{Ga}_{2} \mathrm{As}_{39.2} \mathrm{Se}_{58.8}$ glass. Because of the low concentration of these pyramids and defects in $\mathrm{Ga}_{2} \mathrm{As}_{35.2} \mathrm{Se}_{52.8} \mathrm{Te}_{10}$ glass (altogether $\sim 6 \%$ of the total As sites) they cannot be unambiguously fitted with two separate doublets. While the existence of regular Se-Ass $<(\mathrm{Se})_{2}$ pyramids is expected due to 
the composition, the formation of over-coordinated As is not so obvious. Nevertheless, their nonzero concentration is supported by a tendency in average local coordination number of As, which is slightly higher than three ( 3.1) as obtained from As K-edge EXAFS data analysis for this sample (Table 4). Further increase in Te content leads to the disappearance of this high-BE doublet in the fit of As $3 d$ core level XPS spectra (Table 2). Simultaneously, the average local coordination number for As approaches three (Table 4) as expected from its normal valence state. So, it is unlikely that over-coordinated As atoms can be found in detectable concentration in the structure of Ga-modified TAS, when Te content is higher than 10 at.\%. Most probably, in glasses with higher Te content the As atoms form (Te,As)-As $<(\mathrm{Se})_{2}$ and Se-As $<(\mathrm{Te}, \mathrm{As})_{2}$ pyramidal units containing Te-As or/and As-As bonds, which result in two doublets in the As 3d core level XPS spectra with main components at $\sim 42.0$ and $\sim 41.5 \mathrm{eV}$, respectively (Table 2). The Se atoms in these Te-rich samples have coordination close to two ( 1.8-2.1) as obtained from EXAFS at Se K-edge (Table 4) and, presumably, are bonded with two As or (As,Te)/Ga atoms, forming As-Se-(As,Te) or (As,Te)Se-Ga structural fragments with main components of corresponding doublets at $~ 54.0 \mathrm{eV}$ (Se-I peak) and $\sim 53.3 \mathrm{eV}$ (Se-II peak), respectively. Their moieties, estimated by the area under corresponding doublets (Table 1), agree well with such an assignment. The Se-II peak can include also a component associated with singly-coordinated (terminal, dangling bonds) Se, if present in the structure.

The above assignment of XPS peaks to structural units is fully supported by Raman studies (Fig. 6). The observed vibrational bands for $\mathrm{As}_{40} \mathrm{Se}_{60}$ glass in $200-260 \mathrm{~cm}^{-1}$ region correspond to stretching modes of $\mathrm{AsSe}_{3 / 2}$ pyramidal units and their mutual connection, while those in the range of $90-150 \mathrm{~cm}^{-1}$ are due to various bending modes, whose analysis, however, is much more complicated [35]. With Te addition the stretching modes shift to the low-frequency domain [9], which is expected due to the substitution of one or two Se atoms with heavier Te atoms. They, however, do not fully coincide with the positions and intensities of stretching modes observed for pure $\mathrm{As}_{2} \mathrm{Te}_{3}$ (shown in Fig. 6 for comparison), which is consistent with XPS result on the prevailed formation of mixed Se-As $<(\mathrm{Te}, \mathrm{As})_{2}$ pyramidal units over the pure $\mathrm{AsTe}_{3 / 2}$ pyramids. The exact 
assignment of Raman bands in the low-frequency region demands extensive theoretical calculations and is hindered by the overlap with bending vibrations. The Raman peaks of Ga-based complexes (at $~ 105, ~ 118, ~ 155, ~ 250$ and $\sim 290 \mathrm{~cm}^{-1}$ for $\mathrm{Ga}_{2} \mathrm{Se}_{3}$ ) [36] are hardly observed in the spectra of the investigated samples, because of the low Ga concentration (2 at.\%) and their strong overlap with pyramidal modes. Therefore, information on Ga structural units, which could be obtained from Raman spectroscopy of the investigated materials, is limited.

On the other hand, the compositional behaviour of XPS and EXAFS data for Ga atoms shows remarkable patterns. The first coordination shell fit of EXAFS data recorded at Ga K-edge (Fig. 5, Table 4) of the investigated samples suggests the value for the average local coordination number around Ga close to 4 when there is no Te in the composition, like in the case of other Gamodified selenide glasses [20,21] or $\mathrm{Ga}_{2} \mathrm{Se}_{3}$ crystal [37]. However, the increase in Te content leads to a detectable increase in Ga coordination beyond four (simultaneous changes in Debye-Waller factor are not so significant), which is a somewhat unexpected result (Table 4). It reaches maximum in $\mathrm{Ga}_{2} \mathrm{As}_{31.2} \mathrm{Se}_{46.8} \mathrm{Te}_{20}$ composition, which is the composition with maximum Te content and still fully amorphous XRD pattern [19]. Then, coordination slightly decreases in partially crystallized $\mathrm{Ga}_{2} \mathrm{As}_{27.2} \mathrm{Se}_{40.8} \mathrm{Te}_{30}$ (Table 4), which can be attributed to the preferable tetrahedral coordination of Ga in crystalline compounds with chalcogens [38]. On the other hand, the Ga $3 d$ core level electron XPS spectra can be well fitted with only one doublet with main component $\left(3 d_{5 / 2}\right)$ at $\sim 19.3 \mathrm{eV}$ for all Te-containing glassy samples (Fig. 4 and Table 3). Its FWHM increases and position shifts to the high-BE values ( $19.5 \mathrm{eV}$ ) for the samples with partially crystallized $\mathrm{Ga}_{2} \mathrm{Se}_{3}$ phase (Table 3), which is consistent with other XPS data reported for crystalline $\mathrm{Ga}_{2} \mathrm{Se}_{3}$ [39]. The position and FWHM of Ga 3d core level electron XPS spectra of Te-containing glasses and the increased local coordination number observed with EXAFS at Ga K-edge suggest that additional neighbours appear in the vicinity of Ga, which, however, are not covalently bonded with it (otherwise, increased coordination would cause high-BE shift of Ga 3d core level electron XPS spectrum or appearance of additional doublet at high-BE side [40], but experimentally the opposite shift is observed when compared to the Te-free sample). Most likely, these neighbours are Te atoms in view of the 
compositional dependences of $\mathrm{Ga}$ and Te coordination numbers obtained from the first coordination shell fit of EXAFS data recorded at Ga and Te K-edges (Fig. 5, Table 4). Remarkably, the minimum for the local coordination of Te is observed for the glass composition $\mathrm{Ga}_{2} \mathrm{As}_{31.2} \mathrm{Se}_{46.8} \mathrm{Te}_{20}$ with maximum coordination of Ga. Also, the small values in Te coordination correlate well with the observation of low-BE doublets with the main component at $~ 39.6 \mathrm{eV}$ (Te-II, Table 3) in Te $5 d$ core level electron XPS spectra. All these results, together with the observed increase in the average nearest neighbour distance around Te (Table 4), are consistent with the idea of singly-coordinated (terminal) Te atoms in the vicinity of Ga, and/or changing its bond type from covalent to metallic [41]. The other strong doublet in Te $5 d$ core level electron XPS spectra (Te-I) with main component at $\sim 40.4 \mathrm{eV}$ (Table 3) is, therefore, assigned to the two-fold coordinated Te covalently bonded with As and Se in As-Te-Se complexes. The covalent character of these particular bonds can be additionally inferred from EXAFS data, which show average nearest neighbours distance around Te atoms (Table 4) comparable to the length of covalent bonds in vitreous arsenic tellurides $[41,42]$. Altogether, these results allow us to hypothesize a significant metallic component of bonding in the vicinity of Ga when Te atoms in the glass matrix. Slight decrease in the local coordination of Se below two (Table 4) also favours such an assumption. This effect is consistent with a general metallike behaviour in chalcogenide glasses, e.g. the metals in the amorphous network seek the maximum possible coordination [43].

So, when $\mathrm{Ga}$ is introduced into the $\mathrm{As}_{2} \mathrm{Se}_{3}$ vitreous matrix without Te (Te-free sample) certain number of As atoms in the vicinity of Ga is converted into four-fold coordinated state (or condenses at the grain boundaries if large enough $\mathrm{Ga}_{2} \mathrm{Se}_{3}$ crystallites are formed), causing the appearance of high-BE peak in the As $3 d$ core level electron XPS spectra and a slight increase in the local coordination number beyond three as obtained from EXAFS analysis [18]. With the addition of Te into Ga-modified glass network, Te atoms passivate this mechanism, changing the environment around Ga closer to the dense packing of spheres proper to metallic bonding. As a result, the second neighbour of Te atoms in the vicinity of Ga occurs at very irregular distances, possibly leading to the observed decrease in local coordination number as obtained from EXAFS. 
Such structure can be achieved by the agglomeration of terminal Te atoms in the vicinity of Gaselenide complexes. This would explain the decrease in the average local coordination number of As to $\sim 3$ and slight decrease in Se local coordination as obtained from EXAFS data at corresponding K-edges for samples with Te content (Table 4). In other words, Ga atoms serve as the quenching sites for Te terminal defects, so that rest of the structure can form a fully saturated covalently-bonded network. Existence of terminal Te bonds and over-coordinated As atoms in the structure of TAS glasses was also suggested by Vazquez et al [44], while questioned by Jovari et al [45]. Therefore, the exact physical nature of this phenomenon requires further thorough investigations, specifically in relation to the synthesis conditions.

\section{Conclusions}

The structure of $\mathrm{Ga}_{2}\left(\mathrm{As}_{0.4} \mathrm{Se}_{0.6}\right)_{98-\mathrm{x}} \mathrm{Te}_{\mathrm{x}}$ glass family has been comprehensively investigated with XPS, EXAFS and Raman techniques. Valence band XPS spectra are consistent with those observed earlier for other chalcogenides with comparable concentration of chalcogen atoms, having the same features. The XPS and EXAFS data analysis shows that $~ 90-95 \%$ of As, Se and Te atoms form pyramidal structural units and chalcogen bridges based on covalent bonds, with coordinations of three for arsenic and two for chalcogens. The XPS spectra of Te-containing glasses suggest that almost all pyramids have at least one Te-As or/and As-As bond forming Te-As $<(\mathrm{Se})_{2}$ and SeAs $<(\mathrm{Te}, \mathrm{As})_{2}$ structural units, in agreement with the compositional dependence of Raman spectra. Contrary to the majority of As, Se and Te, the coordination of all Ga atoms is found to be higher than three (that is expected from its main valence state), increasing with Te content. At higher Te concentration some of Te and, possibly, Se atoms in the proximity of Ga sites are thought to form only one covalent bond. We hypothesize that bonding around Ga atoms includes significant metallic component, when Te atoms are present in their vicinity. Thus, the addition of Ga into As-Se matrix alone stimulates the formation of positively charged over-coordinated As defects $\left(\mathrm{As}_{4}{ }^{+}\right)$to compensate the excess of negative charge associated with over-coordinated Ga (local coordination 
4), while simultaneous addition of Ga and Te leads to the agglomeration of Ga selenide complexes with terminal Te atoms, increasing metallic component of their bonds.

\section{Acknowledgment}

The authors acknowledge support for this research funded by Marie-Curie Action FP7-PEOPLE2010-ITN under the GlaCERCo project. Special thanks to Dr. S. Khalid of X18b beamline at NSLS (Brookhaven National Laboratory, USA) for help with EXAFS. The group from Austin Peay State University acknowledges financial support from NSF Grant DMR-1409160.

\section{References}

1. J.S. Sanghera, I.D. Aggarwal, J. Non-Cryst. Solids 256-257 (1999) 6-16.

2. X. Zhang, B. Bureau, C. Boussard-Pledel, J. Lucas, P. Lucas, Chem. Eur. J. 14 (2008) 432-442.

3. P. Lucas, Zh. Yang, M.K. Fah, T. Luo, Sh. Jiang, C. Boussard-Pledel, M.-L. Anne, B. Bureau, Optical Materials Express 3 (8) (2013) 1049.

4. C. Vigreux, E. Barthélémy, L. Bastard, J.-E. Broquin, M. Barillot, S. Ménard, G. Parent, A. Pradel, Opt. Lett. 36(15) (2011) 2922-2924.

5. S. Cui, R. Chahal, Ya. Shpotyuk, C. Boussard, J. Lucas, F. Charpentier, H. Tariel, O. Loreal, V. Nazabal, O. Sire, V. Monbet, Z. Yang, P. Lucas, B. Bureau, Proc. SPIE 8938 (2014) 893805-19.

6. A. Feltz, Amorphous inorganic materials and glasses, VCH Publishers, New York, 1993.

7. Z.U. Borisova, Glassy semiconductors, Plenum Press, New York - London, 1981.

8. B. Bureau, C. Boussard-Pledel, P. Lucas, X. Zhang, J. Lucas, Molecules 14(11) (2009) 43374350.

9. S. Sen, E.L. Gjersing, B.G. Aitken, J. Non-Cryst. Solids 356 (2010) 2083-2088.

10. M. Popescu, Non-crystalline chalcogenides, Kluwer Academic Publishers, New York-BostonDordrecht-London-Moscow, 2002. 
11. D.C. Kaseman, I. Hung, K. Lee, K. Kovnir, Zh. Gan, B. Aitken, S. Sen, J. Phys. Chem. B 119 (2015) 2081-2088.

12. V.S. Shiryaev, J.-L. Adam, X.H. Zhang, C. Boussard-Pledel, J. Lucas, M.F. Churbanov, J. NonCryst. Solids 336 (2004) 113-119.

13. S. Hocde, C. Boussard-Pledel, G. Fonteneau, J. Lucas, Solid State Sci. 3 (2001) 2790284.

14. Ya. Shpotyuk, B. Bureau, C. Boussard-Pledel, V. Nazabal, R. Golovchak, P. Demchenko, I. Polovynko, J. Non-Cryst. Solids 398-399 (2014) 19.

15. B. G. Aitken, C. W. Ponader, R. S. Quimby, Comptes Rendus Chimie 5(12) (2002) 865.

16. Chalcogenide Glasses: Preparation, properties and application, ed. J-L. Adam and X. Zhang, Woodhead Publishing series in Electronic and Optical Materials No.44, 2014.

17. B. J. Eggleton, B. Luther-Davies, K. Richardson, Nature Photonics 5 (2011) 141-148.

18. R. Golovchak, Ya. Shpotyuk, V. Nazabal, C. Boussard-Pledel, B. Bureau, J. Cebulski, H. Jain, J. Chem. Phys. 142 (2015) 184501-1-10.

19. Ya. Shpotyuk, C. Boussard-Pledel, V. Nazabal, R. Chahal, J. Ari, B. Pavlyk, J. Cebulski, J.L. Doualan, B. Bureau, Optical Materials 46 (2015) 228-232.

20. R. Golovchak, L. Calvez, B. Bureau, H. Jain, J. Chemical Physics 139 (2013) 054508(9).

21. R. Golovchak, L. Calvez, E. Petracovschi, B. Bureau, D. Savytskii, H. Jain, Mat. Chem. Phys. 138 (2013) 909-916.

22. R. Golovchak, A. Kovalskiy, A.C. Miller, H. Jain, O. Shpotyuk, Phys. Rev. B 76 (2007) 125208 (7).

23. J.D. Conny, C.J. Powell, Surf. Interface Anal. 29 (2000) 856.

24. J.F. Moulder, W.F. Stickle, P.E. Sobol, K.D. Bomben, in: Handbook of X-ray Photoelectron Spectroscopy, ed. J. Chastein, Perkin-Elmer Corp., Phys. Electr. Div., Eden Prairie, Minnesota, 1992.

25. E.A. Stern, in: X-ray Absorption: Basic Principles of EXAFS, SEXAFS and XANES, ed. D.C. Koningsberger and R. Prins, John Wiley \& Sons, New York, 1988.

26. B. Ravel, M. Newvile, J. Synchrotron Rad. 12 (2005) 537. 
27. A.L. Renninger, B.L. Averbach, Acta Cryst. B 29 (1973) 1583.

28. D. Lübbers, V. Leute, J. Solid State Chem. 43 (1982) 339.

29. S. Kozyukhin, R. Golovchak, A. Kovalskiy, O. Shpotyuk, H. Jain, Semiconductors 45 (4) (2011) 423-426.

30. L. Pauling, The Nature of the Chemical Bond, Cornell Univ. Press, Ithaca, 1960.

31. R. Golovchak, A. Kovalskiy, O. Shpotyuk, H. Jain, Solid State Commun. 165 (2013) 22.

32. H. Hoshino, I. Yamamoto, T. Miyanaga, H. Ikemoto, H. Endo, J. Non-Cryst. Solids 250-252 (1999) 478.

33. D. Vanderbilt, J.D. Joannopoulos, Phys. Rev. B 23 (1981) 2596.

34. S. Ravindren, K. Gunasekera, Z. Tucker, A. Diebold, P. Boolchand, M. Micoulaut, J. Chem. Phys. 140 (2014) 134501.

35. H. Hahn, W. Klingler, Z. Anorg. Allg. Chem. 259 (1949) 135-142.

36. R. Golovchak, J. Oelgoetz, M. Vlcek, A. Esposito, A. Saiter, J.-M. Saiter, H. Jain, J. Chem. Phys. 140 (2014) 054505.

37. R.G. Valeev, V.V. Kriventsov, N.A. Mezentsev, Bull. Russ. Acad. Sci. Physics 77, 9 (2013) 1154.

38. M. von der Emde, D.R.T. Zahn, T. Ng, N. Maung, G.H. Fan, I.B. Poole, J.O. Williams, A.C. Wright, Applied Surface Science 104/105 (1996) 575-579.

39. H. Iwakuro, C. Tatsuyama, S. Ichimura, Japanese J. Appl. Physics 21 (1982) 94.

40. R. Golovchak, O. Shpotyuk, A. Kovalskiy, A.C. Miller, J. Cech, H. Jain, Phys. Rev. B 77 (2008) 172201(4).

41. J. Cornet, D. Rossier, J. Non-Cryst. Sol. 12 (1973) 85-99.

42. S. Sen, S. Joshi, B.G. Aitken, S. Khalid. Journal of Non-Cryst. Solids 354 (2008) 4620-4625.

43. Z.U. Borisova, E.A. Bychkov, Yu.S. Tveryanovich, Interaction of metals with chalcogenide glasses, Izdatelstvo Leningradskogo Universiteta, Leningrad, 1991.

44. J. Vazquez, P. Villares, R. Jimenez-Garay, J. Non-Cryst. Sol. 86 (1986) 251-260. 
45. P. Jóvári, B. Bureau, I. Kaban, V. Nazabal, B. Beuneu, U. Rütt, J. Alloys and Compounds 488 2009) 39-43. 


\section{Figure captions}

Figure 1. Valence band XPS spectra of $\mathrm{Ga}_{2}\left(\mathrm{As}_{0.4} \mathrm{Se}_{0.6}\right)_{98-\mathrm{x}} \mathrm{Te}_{\mathrm{x}}$ samples.

Figure 2. Fitting of Se $3 d$ core-level spectra for $\mathrm{Ga}_{2}\left(\mathrm{As}_{0.4} \mathrm{Se}_{0.6}\right)_{98-\mathrm{x}} \mathrm{Te}_{\mathrm{x}}$ samples (bold - experimental spectrum, thin - fitted spectrum, dot - base line, dash - fitted components).

Figure 3. Fitting of As $3 d$ and Te $5 d$ core-level spectra for $\mathrm{Ga}_{2}\left(\mathrm{As}_{0.4} \mathrm{Se}_{0.6}\right)_{98-\mathrm{x}} \mathrm{Te}_{\mathrm{x}}$ samples (bold experimental spectrum, thin - fitted spectrum, dot - base line, dash - fitted components: As - red, Te - blue).

Figure 4. Fitting of Ga $3 d$ core-level spectra for $\mathrm{Ga}_{2}\left(\mathrm{As}_{0.4} \mathrm{Se}_{0.6}\right)_{98-\mathrm{x}} \mathrm{Te}_{\mathrm{x}}$ samples (bold - experimental spectrum, thin - fitted spectrum, dot - base line, dash - fitted components).

Figure 5. The $k$-weighted $\chi(k)$ EXAFS oscillations (left panel) and their Fourier Transform (right panel) for the investigated $\mathrm{Ga}_{2}\left(\mathrm{As}_{0.4} \mathrm{Se}_{0.6}\right)_{98-\mathrm{x}} \mathrm{Te} \mathrm{e}_{\mathrm{x}}$ samples at Se (a), As (b), Ga (c) and Te (d) Kedges. Experimental points are shown with symbols, while solid curves represent their best fits.

Figure 6. Raman spectra of the investigated materials. 
Table 1. Numerical parameters ( $3 d_{5 / 2}$ components) of Se $3 d$ core level XPS spectra. BE and fwhm in $\mathrm{eV}, A$ in $\%$.

\begin{tabular}{lcccccc}
\hline core level & \multicolumn{3}{l}{ Se (I): } & \multicolumn{5}{c}{ Se(II): } \\
\cline { 2 - 7 } sample & BE & fwhm & A & BE & fwhm & A \\
& & & & & & \\
\hline As $_{\mathbf{4 0}} \mathbf{S e}_{\mathbf{6 0}}$ & 54.09 & 0.77 & 100 & & & \\
\hline $\mathbf{G a}_{\mathbf{2}} \mathbf{A s}_{\mathbf{3 9 . 2}} \mathbf{S e}_{\mathbf{5 8 . 8}}$ & 54.03 & 0.79 & 96 & 53.30 & 0.84 & 4 \\
\hline $\mathbf{G a}_{\mathbf{2}} \mathbf{A s}_{\mathbf{3 5 . 2}} \mathbf{S e}_{\mathbf{5 2 . 8}} \mathbf{T e}_{\mathbf{1 0}}$ & 53.95 & 0.73 & 97 & 53.21 & 0.67 & 3 \\
\hline $\mathbf{G a}_{\mathbf{2}} \mathbf{A s}_{\mathbf{3 3 . 2}} \mathbf{S e}_{\mathbf{4 9 . 8}} \mathbf{T e}_{\mathbf{1 5}}$ & 53.97 & 0.72 & 97 & 53.22 & 0.70 & 3 \\
\hline $\mathbf{G a}_{\mathbf{2}} \mathbf{A s}_{\mathbf{3 1 . 2}} \mathbf{S e}_{\mathbf{4 6 . 8}} \mathbf{T e}_{\mathbf{2 0}}$ & 53.94 & 0.73 & 93 & 53.25 & 0.63 & 7 \\
\hline $\mathbf{G a}_{\mathbf{2}} \mathbf{A s}_{\mathbf{2 7 . 2}} \mathbf{S e}_{\mathbf{4 0 . 8}} \mathbf{T e}_{\mathbf{3 0}} \mathbf{c r y s t}$ & 54.00 & 0.72 & 94 & 53.28 & 0.86 & 6 \\
\hline
\end{tabular}


Table 2. Numerical parameters ( $3 d_{5 / 2}$ components) of As $3 d$ core level XPS spectra. BE and fwhm in $\mathrm{eV}, \mathrm{A}$ in $\%$.

\begin{tabular}{|c|c|c|c|c|c|c|c|c|c|c|c|c|}
\hline \multirow{2}{*}{$\begin{array}{l}\text { core level } \\
\text { sample }\end{array}$} & \multicolumn{3}{|l|}{$\operatorname{As}(\mathrm{I}):$} & \multicolumn{3}{|c|}{ As (II): } & \multicolumn{3}{|c|}{ As (III): } & \multicolumn{3}{|c|}{ As(IV): } \\
\hline & $\mathrm{BE}$ & fwhm & $\mathrm{A}$ & $\mathrm{BE}$ & Fwhm & $\mathrm{A}$ & $\mathrm{BE}$ & fwhm & $\mathrm{A}$ & $\mathrm{BE}$ & fwhm & $\mathrm{A}$ \\
\hline $\mathrm{As}_{40} \mathrm{Se}_{60}$ & & & & 42.21 & 0.71 & 100 & & & & & & \\
\hline $\mathrm{Ga}_{2} \mathrm{As}_{39.2} \mathrm{Se}_{58.8}$ & 42.36 & 0.45 & 5 & 42.20 & 0.70 & 82 & & & & 41.73 & 0.85 & 13 \\
\hline $\mathrm{Ga}_{2} \mathrm{As}_{35.2} \mathrm{Se}_{52.8} \mathrm{Te}_{10}$ & 42.29 & 0.45 & 6 & & & & 42.05 & 0.63 & 85 & 41.47 & 0.65 & 9 \\
\hline $\mathrm{Ga}_{2} \mathrm{As}_{33.2} \mathrm{Se}_{49.8} \mathrm{Te}_{15}$ & & & & & & & 42.05 & 0.65 & 88 & 41.47 & 0.67 & 12 \\
\hline $\mathrm{Ga}_{2} \mathrm{As}_{31.2} \mathrm{Se}_{46.8} \mathrm{Te}_{20}$ & & & & & & & 42.05 & 0.62 & 72 & 41.62 & 0.83 & 28 \\
\hline $\mathrm{Ga}_{2} \mathrm{As}_{27.2} \mathrm{Se}_{40.8} \mathrm{Te}_{30}$ & & & & & & & 42.09 & 0.62 & 71 & 41.68 & 0.85 & 29 \\
\hline cryst & & & & & & & & & & & & \\
\hline
\end{tabular}


Table 3. Numerical parameters ( $3 d_{5 / 2}$ components) of Ga $3 d$ and Te $5 d$ core level XPS spectra. BE and fwhm in $\mathrm{eV}, A$ in \%.

\begin{tabular}{|c|c|c|c|c|c|c|c|c|c|}
\hline \multirow{2}{*}{$\begin{array}{l}\text { core level } \\
\text { sample }\end{array}$} & \multicolumn{3}{|l|}{ Ga(I): } & \multicolumn{3}{|l|}{ Te (I): } & \multicolumn{3}{|c|}{ Te (II): } \\
\hline & $\mathrm{BE}$ & fwhm & $\mathrm{A}$ & $\mathrm{BE}$ & fwhm & $\mathrm{A}$ & $\mathrm{BE}$ & fwhm & $\mathrm{A}$ \\
\hline $\mathrm{Ga}_{2} \mathrm{As}_{39.2} \mathrm{Se}_{58.8}$ & 19.41 & 0.89 & 100 & & & & & & \\
\hline $\mathrm{Ga}_{2} \mathrm{As}_{35.2} \mathrm{Se}_{52.8} \mathrm{Te}_{10}$ & 19.26 & 0.77 & 100 & 40.41 & 0.86 & 100 & & & \\
\hline $\mathrm{Ga}_{2} \mathrm{As}_{33.2} \mathrm{Se}_{49.8} \mathrm{Te}_{15}$ & 19.29 & 0.79 & 100 & 40.40 & 0.81 & 98 & 39.55 & 0.42 & 2 \\
\hline $\mathrm{Ga}_{2} \mathrm{As}_{31.2} \mathrm{Se}_{46.8} \mathrm{Te}_{20}$ & 19.24 & 0.82 & 100 & 40.37 & 0.81 & 95 & 39.56 & 0.62 & 5 \\
\hline $\mathrm{Ga}_{2} \mathrm{As}_{27.2} \mathrm{Se}_{40.8} \mathrm{Te}_{30}$ cryst & 19.45 & 1.12 & 100 & 40.41 & 0.76 & 97 & 39.56 & 0.52 & 3 \\
\hline
\end{tabular}


Table 4. Fitting parameters for Ga, As and Se K-edges Fourier transformed EXAFS spectra for $\mathrm{Ga}_{2}\left(\mathrm{As}_{0.4} \mathrm{Se}_{0.6}\right)_{98-\mathrm{x}} \mathrm{Te}_{\mathrm{x}}$ glasses ( $R$ is the distance from neighbouring atom to the absorbing atom; $\sigma_{0}^{2}$ is Debye-Waller factor and $N_{j}$ is the local coordination number).

\begin{tabular}{|c|c|c|c|c|c|c|c|c|c|c|c|c|}
\hline \multirow{2}{*}{$\begin{array}{r}\text { K-edge } \\
\text { composition }\end{array}$} & \multicolumn{3}{|c|}{ Ga } & \multicolumn{3}{|c|}{ As } & \multicolumn{3}{|c|}{ Se } & \multicolumn{3}{|c|}{ Te } \\
\hline & $\begin{array}{l}\boldsymbol{R}, \AA \\
( \pm 0.01)\end{array}$ & $\begin{array}{l}\sigma_{0}^{2}, \AA^{2} \\
( \pm 0.0007)\end{array}$ & $\begin{array}{l}N_{G a} \\
( \pm 0.5)\end{array}$ & $\begin{array}{l}\boldsymbol{R}, \boldsymbol{A} \\
( \pm 0.01)\end{array}$ & $\begin{array}{l}\sigma_{0}^{2}, \AA^{2} \\
( \pm 0.0005)\end{array}$ & $\begin{array}{l}N_{A s} \\
( \pm 0.5)\end{array}$ & $\begin{array}{l}\boldsymbol{R}, \boldsymbol{A} \\
( \pm 0.01)\end{array}$ & $\begin{array}{l}\sigma_{0}^{2}, \AA^{2} \\
( \pm 0.0005)\end{array}$ & $\begin{array}{l}N_{S e} \\
( \pm 0.5)\end{array}$ & $\begin{array}{l}\boldsymbol{R}, \boldsymbol{A} \\
( \pm 0.01)\end{array}$ & $\begin{array}{l}\sigma_{0}^{2}, \AA^{2} \\
( \pm 0.0007)\end{array}$ & $\begin{array}{l}\boldsymbol{N}_{T e} \\
( \pm 0.5)\end{array}$ \\
\hline $\mathrm{As}_{40} \mathrm{Se}_{60}$ & & & & 2.42 & 0.0053 & 3.0 & 2.42 & 0.0051 & 2.0 & & & \\
\hline $\mathrm{Ga}_{2} \mathrm{As}_{39.2} \mathrm{Se}_{58.8}$ & 2.40 & 0.0061 & 4.0 & 2.42 & 0.0052 & 3.2 & 2.42 & 0.0050 & 2.1 & & & \\
\hline $\mathrm{Ga}_{2} \mathrm{As}_{35.2} \mathrm{Se}_{52.8} \mathrm{Te}_{10}$ & 2.41 & 0.0061 & 4.3 & 2.43 & 0.0052 & 3.1 & 2.42 & 0.0045 & 1.9 & 2.65 & 0.0058 & 2.0 \\
\hline $\mathbf{G a}_{2} \mathbf{A s}_{33.2} \mathbf{S e}_{49.8} \mathbf{T e}_{15}$ & 2.42 & 0.0062 & 4.4 & 2.44 & 0.0051 & 3.0 & 2.42 & 0.0045 & 1.9 & 2.67 & 0.0048 & 1.7 \\
\hline $\mathbf{G a}_{2} \mathrm{As}_{31.2} \mathrm{Se}_{46.8} \mathrm{Te}_{20}$ & 2.42 & 0.0061 & 5.2 & 2.43 & 0.0053 & 3.0 & 2.42 & 0.0045 & 1.8 & 2.69 & 0.0034 & 1.4 \\
\hline $\begin{array}{l}\mathbf{G a}_{2} \mathrm{As}_{27.2} \mathrm{Se}_{40.8} \mathrm{Te}_{30} \\
\text { cryst }\end{array}$ & 2.43 & 0.0068 & 4.8 & 2.44 & 0.0055 & 3.0 & 2.43 & 0.0042 & 1.8 & 2.70 & 0.0042 & 1.6 \\
\hline
\end{tabular}




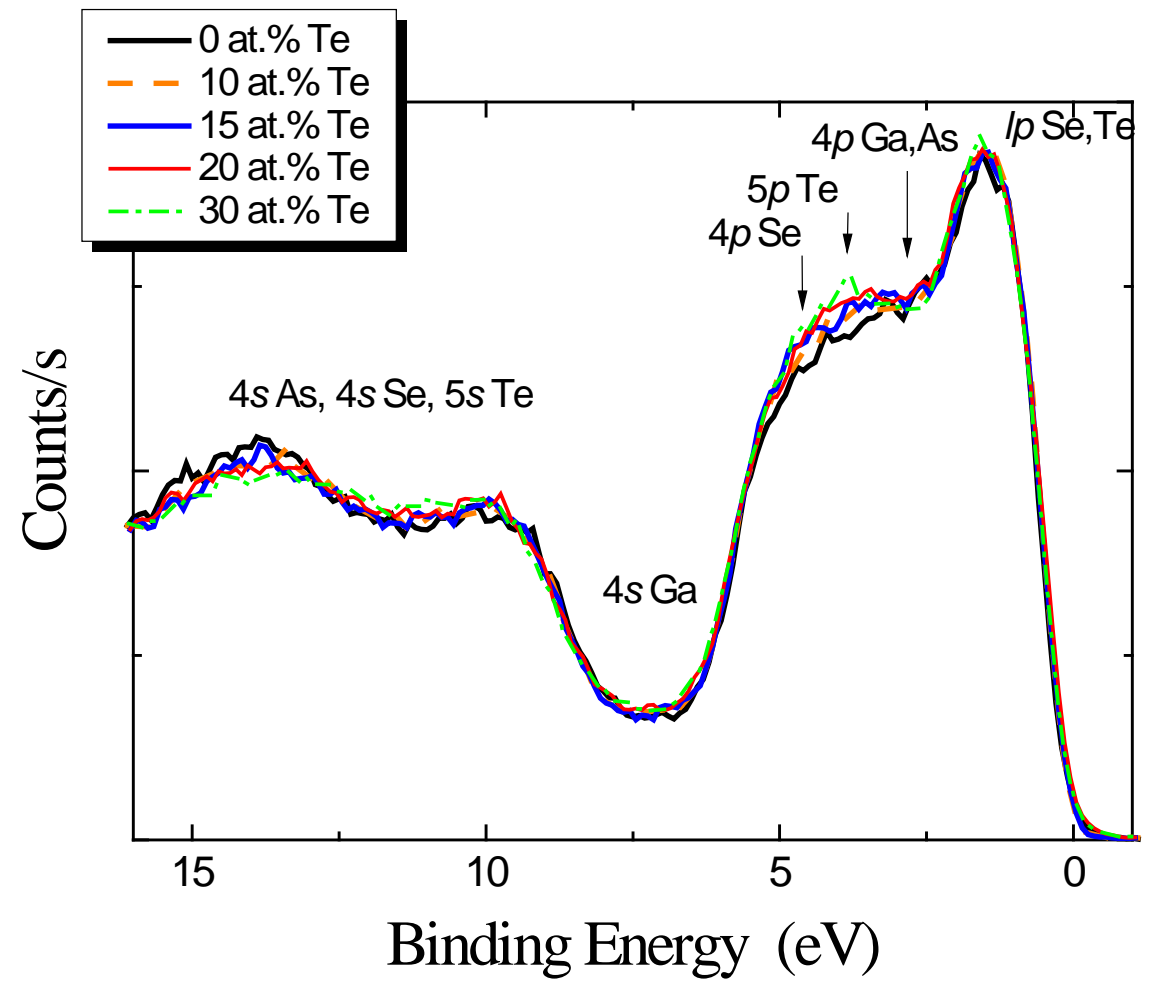

Figure 1. 

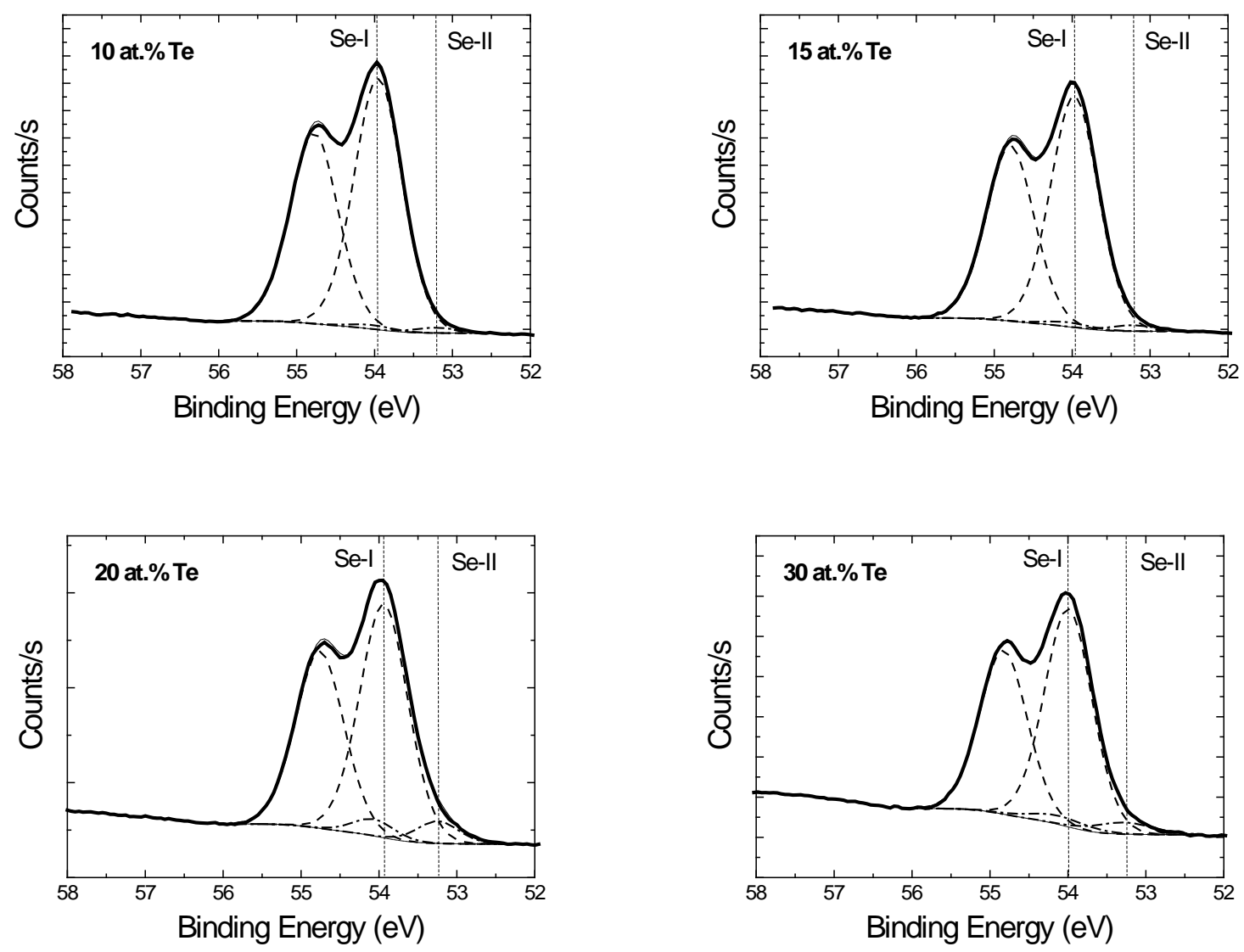

Figure 2. 

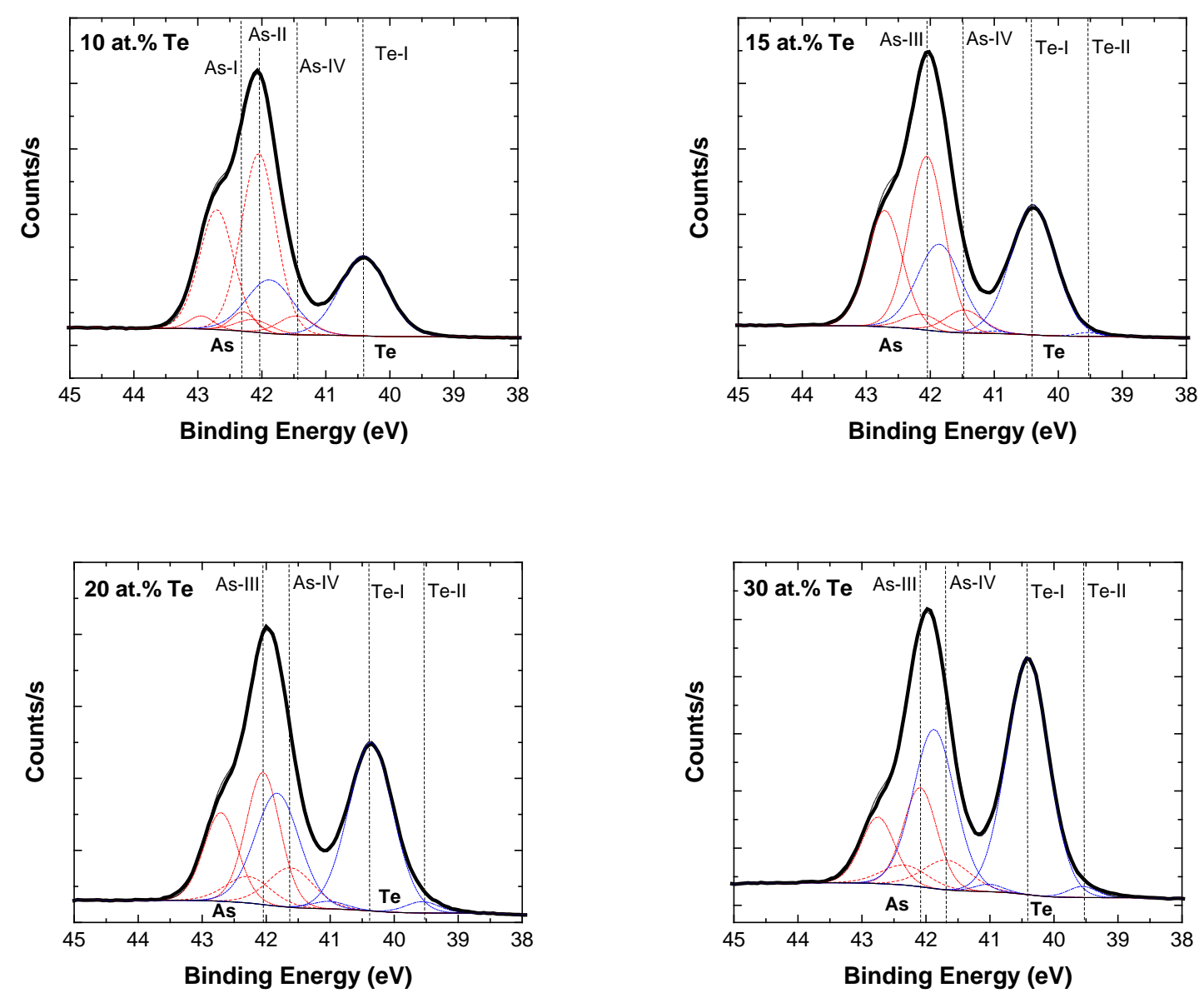

Figure 3. 

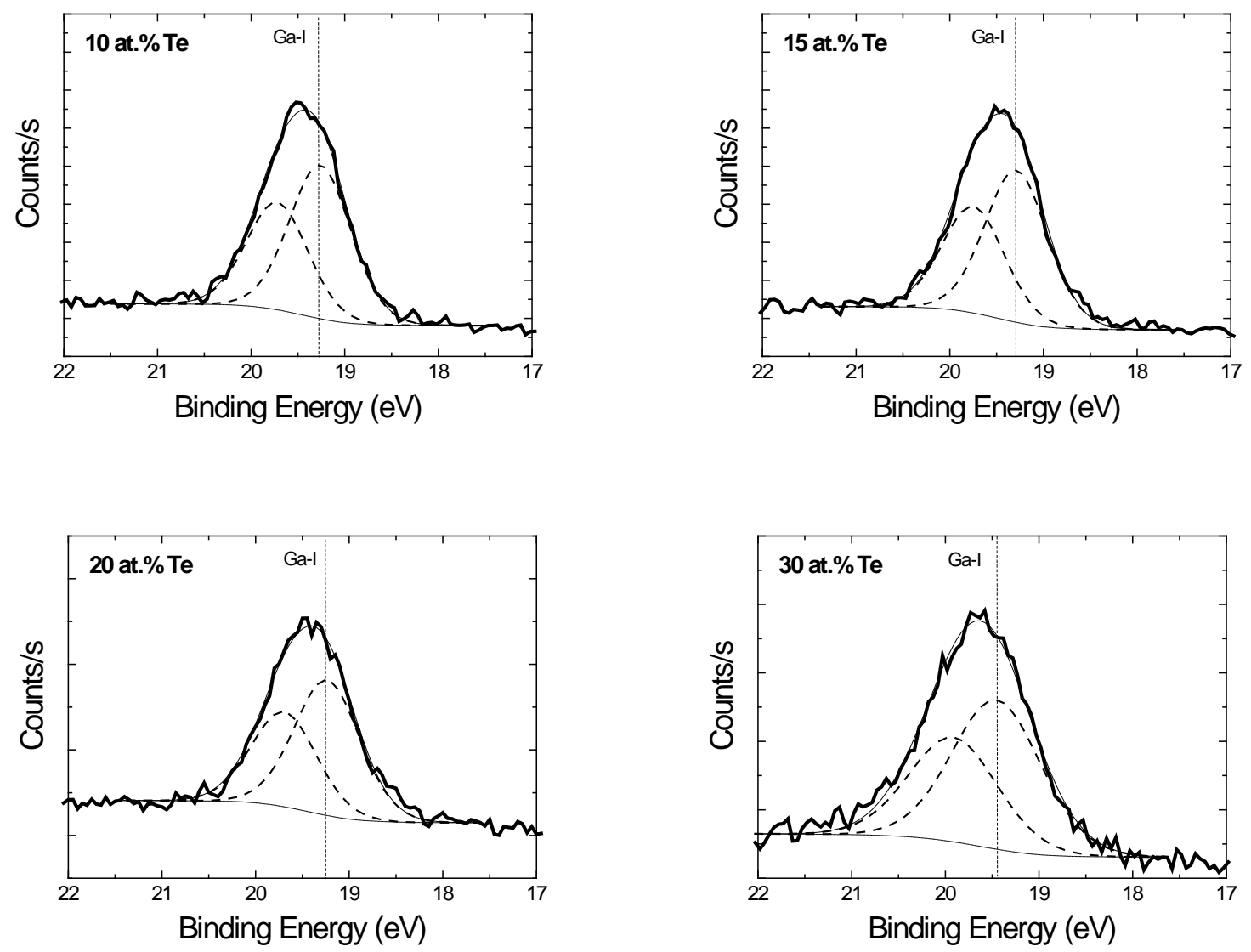

Figure 4.

(a)
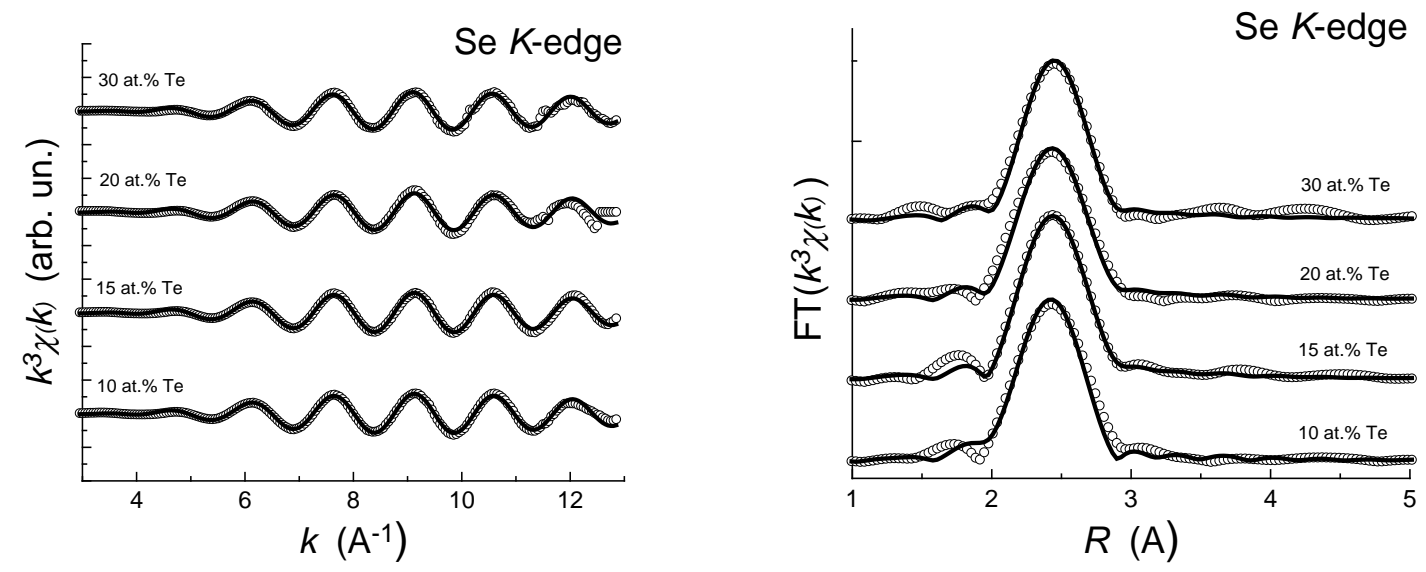
(b)


(c)

Te K-edge
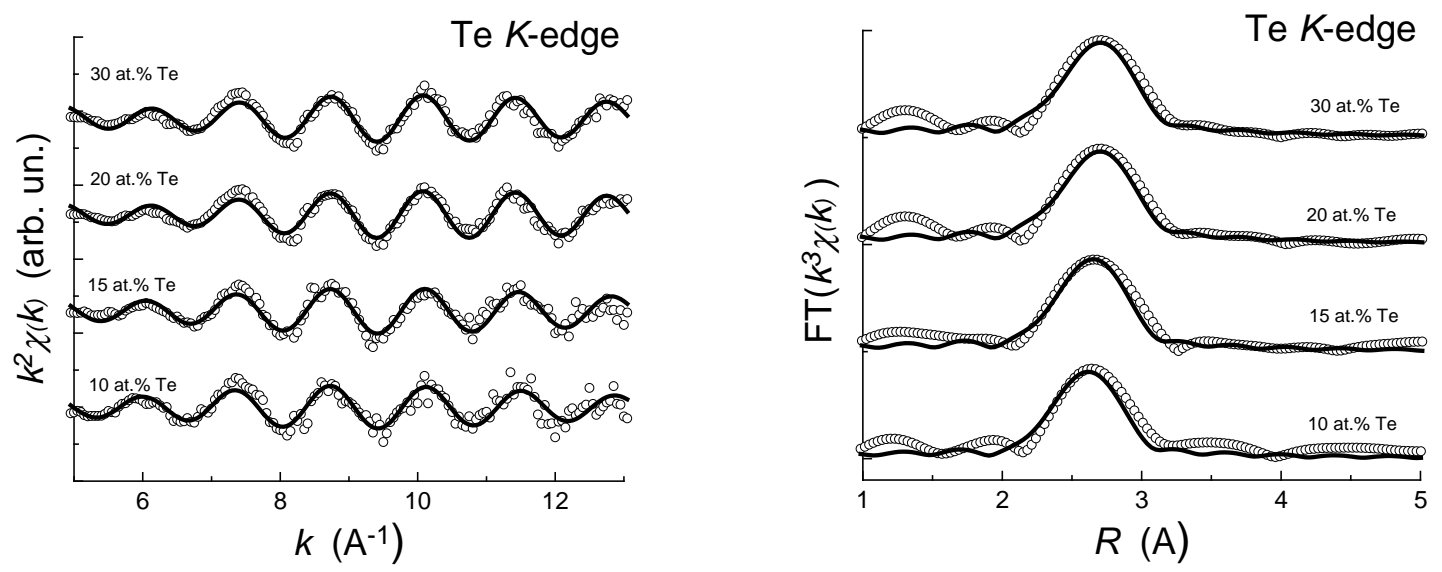

Figure 5. 


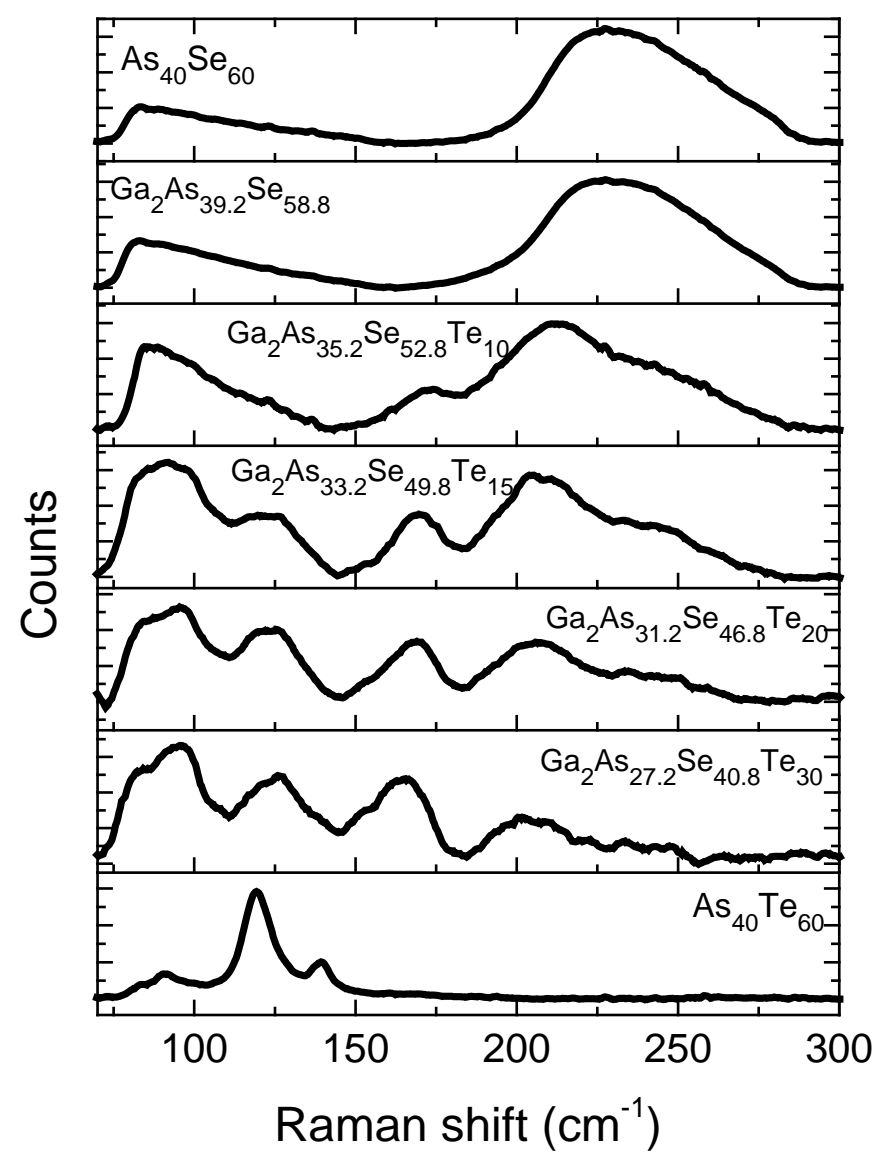

Figure 6. 